\title{
A Full MALDI-Based Approach to Detect Plasmid-Encoded KPC-Producing Klebsiella pneumoniae
}

\begin{abstract}
Miriam Cordovana ${ }^{*}$, Markus Kostrzewa², Jörg Glandorf², Michael Bienia ${ }^{3}$, Simone Ambretti ${ }^{1}$ and Arthur B. Pranada ${ }^{3}$
\end{abstract}

'Laboratory of Bacteriology, Operative Unit of Microbiology, University Hospital of Bologna Policlinico Sant'Orsola-Malpighi, Bologna, Italy, ${ }^{2}$ Bruker Daltonik GmbH, Bremen, Germany, ${ }^{3}$ Department of Medical Microbiology, MVZ Dr. Eberhard \& Partner Dortmund, Dortmund, Germany

KPC-producing Klebsiella pneumoniae represents a severe public health concern worldwide. The rapid detection of these isolates is of fundamental importance for the adoption of proper antibiotic treatment and infection control measures, and new applications of MALDI-TOF MS technology fit this purpose. In this study, we present a full MALDI-based approach to detect plasmid-encoded KPC-producing strains, accomplished by the automated detection of a KPC-specific peak (at 11,109 m/z) by a specific algorithm integrated into the MALDI Biotyper system (Bruker Daltonik), and the confirmation of carbapenemase activity by STAR-Carba imipenem hydrolysis assay. A total of 6209 K. pneumoniae isolates from Italy and Germany were investigated for the presence of the KPC-related peak, and a subset of them $(n=243)$ underwent confirmation of carbapenemase activity by STAR-Carba assay. The novel approach was further applied directly to positive blood culture bottles $(n=204)$, using the bacterial pellet obtained with Sepsityper kit (Bruker Daltonik). The novel approach enabled a reliable and very fast detection of KPC-producing $K$. pneumoniae strains, from colonies as well as directly from positive blood cultures. The automated peak detection enabled the instant detection of KPC-producing $K$. pneumoniae during the routine identification process, with excellent specificity (100\%) and a good sensitivity (85.1\%). The sensitivity is likely mainly related to the prevalence of the specific plasmid harboring clones among all the KPC-producing circulating strains. STAR-Carba carbapenemase confirmation showed $100 \%$ sensitivity and specificity, both from colonies and from positive blood cultures.

Keywords: MALDI-TOF MS, KPC, Klebsiella pneumoniae, carbapenemase, multidrug resistance, KPC-related peak, pKpQIL plasmid

\section{INTRODUCTION}

Carbapenem-resistant Enterobacteriaceae (CRE) have recently emerged as a class of bacterial pathogens that threaten the effectiveness of last resort treatment, posing a serious threat to global public health (Tzouvelekis et al., 2012). Even though resistance to carbapenems may involve several combined mechanisms (Nordmann et al., 2012a), from the public health point of view 
attention is focused on the isolates that produce specific carbapenem-hydrolyzing $\beta$-lactamases (carbapenemases). Such resistance determinants can be transferred between bacteria of the same or of different species by mobile genetic elements (Kumarasamy et al., 2010; Nordmann et al., 2012b). The carbapenemase transmission often is linked to other non$\beta$-lactam resistance determinants, leading to the rise and the rapid dissemination of multi-drug or pan-drug resistant (MDR, PDR) organisms (Kumarasamy et al., 2010).

Carbapenemase-producing Enterobacteriaceae (CPE) have become important causes of hospital acquired infections, associated with a significantly increased mortality, especially in critical wards (Vincent et al., 2009). In Europe, the highest prevalences have been reported from Greece, with $61.9 \%$ of carbapenem-resistant $K$. pneumoniae among invasive isolates, Italy with $33.5 \%$, and from Romania (24.7\%) (EARS-Net report 2016).

CPE show an epidemiology according to the enzyme type, with different prevalence in the different geographic areas. In Europe, KPC-producing K. pneumoniae is endemic in Italy and Greece, while in other countries class D (OXA-48 family) and class $\mathrm{B}(\mathrm{M} \beta \mathrm{L}$ - Metallo- $\beta$-Lactamases) carbapenemases are reported with overall lower prevalence. Nevertheless, the Klebsiella pneumoniae Carbapenemase (KPC) family has the most extensive global distribution of all carbapenemases associated with Enterobacterales (van Duin and Doi, 2017), and are the most clinically significant (Munoz-Price et al., 2013). KPCs are found in many Gram-negative species, including both Enterobacterales and non-fermenters, but $K$. pneumoniae is the most largely predominant species.

Actually, 20 variants of the blaKPC gene have been described, and the variants KPC-2 and KPC-3 are the most common (Doumith et al., 2017). The bla KPC genes have been identified in a variety of plasmids (Chen et al., 2014), and reported in more than 100 different Sequence Types (ST) of K. pneumoniae. The transmission of bla $a_{\mathrm{KPC}}$ genes can be mediated by different molecular mechanisms, from mobility of small genetic elements (i.e., Tn4401 transposon) to horizontal transfer of plasmids, and via clonal spread (Munoz-Price and Quinn, 2009; Partridge, 2014). Nevertheless, the global diffusion of plasmid-borne bla $a_{\mathrm{KPC}}$ has been linked to the clonal dissemination worldwide of a major multilocus sequence type (MLST or ST), namely ST258, and its related variants (Cuzon et al., 2010; Munoz-Price et al., 2013). KPC-Kp are considered endemic in several countries, including the North-eastern United States, Argentina, Brazil, Puerto Rico, Colombia, China, Israel, and, in Europe, Italy and Greece (Munoz-Price et al., 2013; Nordmann and Poirel, 2014). As treatment options are very limited and because of the epidemiological impact, rapid methods to detect CPE, and differentiate them from other CRE are desired. Several techniques have been developed, relying on different molecular or phenotypical principles. Although proven to be effective for the detection of KPC-producing isolates, overall these methods are either expensive and limited in the targets included, or slow with a time to report up to $24 \mathrm{~h}$, and lack in sensitivity and/or specificity (Osei Sekyere et al., 2015; Bialvaei et al., 2016; Lutgring and Limbago, 2016; Rood and Li, 2017; Tamma et al., 2017).
Recently, it has been shown that the intrinsic speed of MALDI-TOF MS technology could be deployed and successfully applied to a prompt detection of carbapenemase-producing strains. First, it was proven that the hydrolytic activity of carbapenemases can be detected by a functional assay that relies on the evaluation of the mass spectra of the carbapenem molecule after a short incubation with the bacterial strains (Burckhardt and Zimmermann, 2011; Sparbier et al., 2012; Hrabák et al., 2013; Johansson et al., 2014; Rapp et al., 2018). This is even possible directly from positive blood cultures (Jung et al., 2014; Hoyos-Mallecot et al., 2014; Sakarikou et al., 2017).

Further, Lau et al. (2014) discovered a peak in MALDITOF mass spectra of KPC-producing $K$. pneumoniae related to a $\mathrm{pKpQIL}$ plasmid carrying bla $a_{\mathrm{KPC}}$. This specific peak at $11,109 \mathrm{~m} / \mathrm{z}$ is clearly detectable in bacterial MALDI-TOF mass spectra. Different approaches to seek for this peak have been used and evaluated (Lau et al., 2014; Gaibani et al., 2016; Youn et al., 2016). Until recently, the detection of this peak required a manual operation, with visual analysis or additional software in a second step after the routine identification process, and therefore impeding a real-time detection of KPC strains (Lau et al., 2014; Gaibani et al., 2016; Youn et al., 2016).

In this study, we present a full MALDI-based approach to an instant detection of KPC-producing K. pneumoniae strains simultaneously during the standard routine species identification process. KPC-producers were indirectly identified by the automated detection of a specific peak related to a bla $a_{\mathrm{KPC}}$-carrying $\mathrm{pKpQIL}$ plasmid, using an algorithm that was integrated into the MALDI Biotyper software. A MALDI-TOF based imipenem hydrolysis assay was further used to confirm KPC enzyme activity.

This novel approach was applied to well characterized strains, to a large set of clinical routine strains, and finally directly to routine positive blood cultures.

\section{MATERIALS AND METHODS}

\section{Optimization of the Algorithm for Automated KPC Detection With PCR Confirmed Strains}

For automated detection of the pKpQIL related peak described by Lau et al. (2014), a software algorithm was developed. The peak detection was based on a manual analysis of spectra analyzed with the flexAnalysis software version 3.4 (Bruker Daltonik, Bremen, Germany) - Figure 1. Spectra were normalized and smoothed with standard settings. For precise detection the spectra were screened for potential peaks for an internal recalibration as described in Pranada et al. (2017). After internal recalibration, intensities 3 times higher than the surrounding noise were then counted as peaks. If such potential peak was detected in a window $\pm 5 \mathrm{~m} / \mathrm{z}$ around the previously described mass of $11,109 \mathrm{~m} / \mathrm{z}$, the algorithm yielded the detection of the KPC related peak. Examples of these automated detections are shown in Figure 2. 


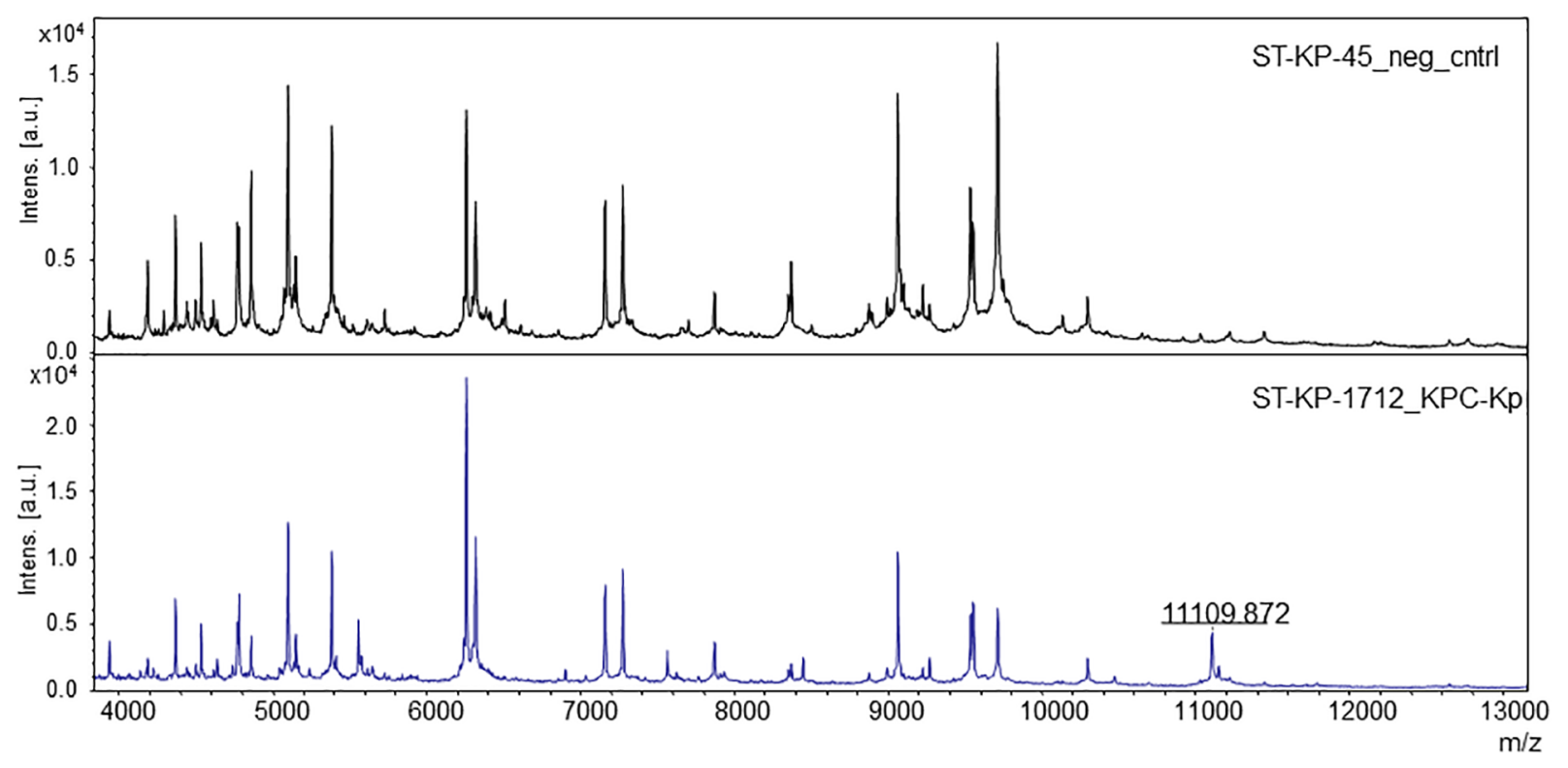

FIGURE 1 | The pKpQIL plasmid-related peak in the MALDI mass spectra of $K$. pneumoniae. The upper spectrum shows a negative control, without the specific peak. The lower spectrum shows a KPC-producing strain exhibiting the specific peak.

$N=266$ spectra of $K$. pneumoniae previously characterized by PCR (Hyplex ${ }^{\circledR}$ SuperBug ID assay - Amplex Biosystems GmbH, Gießen, Germany), were used to test the algorithm. Among them, $n=152$ were KPC-producers, and $n=114$ were negative for the bla $a_{\mathrm{KPC}}$ gene.

To assess the technical sensitivity of the automatic detection, all the spectra were also analyzed by visual inspection using the FlexAnalysis software (Bruker Daltonik GmbH) to seek for the presence of the KPC-specific peak.

\section{Evaluation of the Automated KPC Detection With a Large Collection of Strains}

The optimized algorithm was integrated into the commercial MALDI Biotyper RUO software, and the method was evaluated on a large set of clinical strains from Italy (in the Microbiology Unit of the University Hospital of Bologna Policlinico Sant'Orsola-Malpighi), as a country with a high prevalence of KPC-bearing strains, and from Germany (in the Department of Microbiology of the MVZ Dr. Eberhard \& Partner Dortmund, Dortmund, Germany) as a low prevalence country.

A total of $n=6209$ MALDI-TOF mass spectra of clinical and surveillance isolates of $K$. pneumoniae collected in a time frame between 2009 and 2017 were analyzed. The spectra were retrieved from the database of stored runs from routine identification in Dortmund. In Bologna, spectra for strains isolated between 2010 and September 2016 were recorded retrospectively from the collection of frozen strains as well as prospectively from October 2016 to December 2017.

Bacterial strains were cultured for 24-48 h on Tryptose Soy Agar with sheep blood in Bologna, and on Columbia Agar with sheep blood in Dortmund. Routine susceptibility testing was performed using VITEK2 XL systems (bioMérieux, Marcy l'Étoile, France).

All isolates with MICs greater than the EUCAST epidemiological cut-off for at least one of the carbapenems (ertapenem, meropenem, imipenem) underwent either detection of carbapenemase-encoding genes (in Dortmund by an inhouse PCR) or confirmation of carbapenemase production, (in Bologna by disk diffusion synergy test with inhibitors (KPC+MBL Confirm ID Pack, ROSCO Diagnostika, Taastrup, Denmark), immunochromatographic assay (OXA-48 K-Set, CorisBioConcept, Gembloux, Belgium), and/or PCR (Hyplex ${ }^{\circledR}$ SuperBug ID assay -Amplex Biosystems GmbH, Gießen, Germany, GeneXpert ${ }^{\circledR}$ Carba-R -Cepheid, Sunnyvale, CA, United States).

Among these strains, at routine testing $n=2390$ were KPCproducers ( $n=2385$ from Bologna, $n=5$ from Dortmund), $n=179 \mathrm{M} \beta \mathrm{L}$-producers $(n=119$ from Bologna, $n=60$ from Dortmund), $n=32$ OXA-48 family producers ( $n=19$ from Bologna, $n=13$ from Dortmund), $n=221$ were negative for carbapenemase production but resistant to carbapenems (likely ES $\beta L / A m p C$ producers associated with reduced membrane permeability $-n=207$ from Bologna, $n=14$ from Dortmund), and $n=3387$ susceptible to carbapenems ( $\beta$-lactamases producers or wild type $-n=105$ from Bologna, $n=3282$ from Dortmund) - Table 1.

\section{Evaluation of Stability and Reliability of KPC-Peak Detection}

Further, the sensitivity of the automated KPC detection by MALDI-TOF MS was assessed with regard to the type of culture medium and the age of cultures: spectra of $n=34$ KPCproducing strains cultured on Sheep Blood Agar, CHR-KPC Agar, 
A

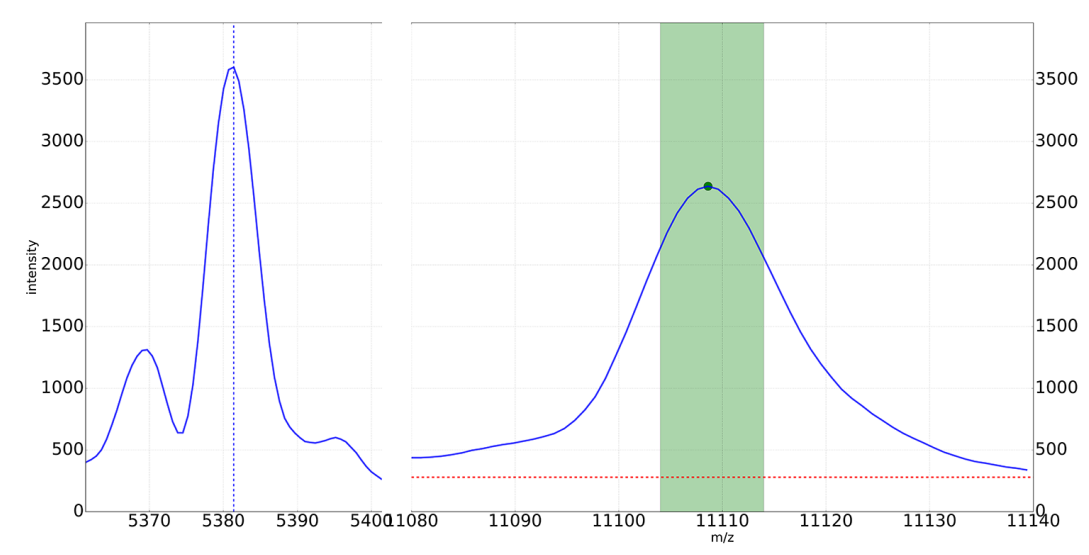

B

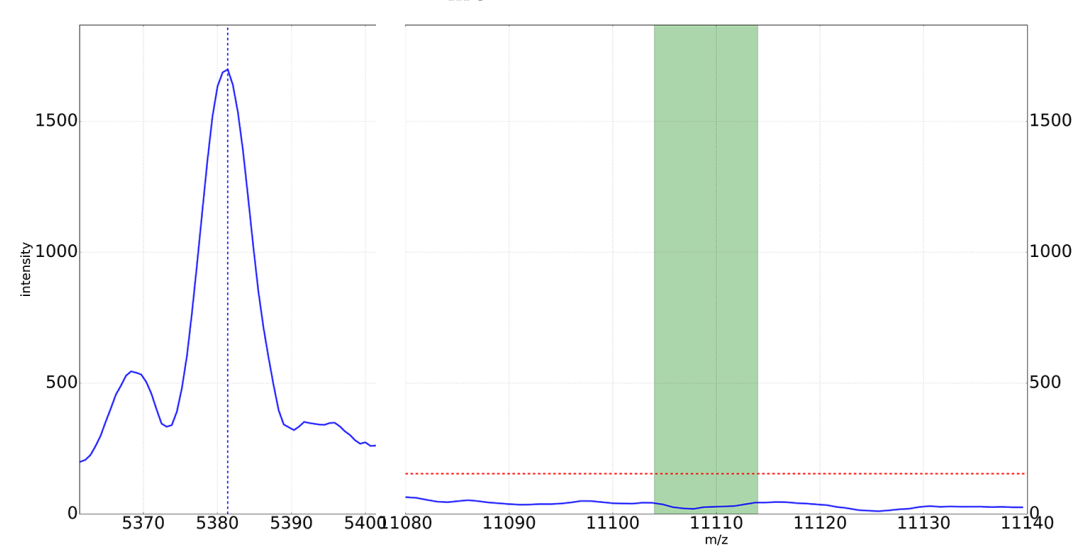

FIGURE 2 | Detection of the KPC-related peak by the automated algorithm. An internal calibration peak specific to $K$. pneumoniae is recognized and used for higher precision in peak detection in the window of $\mathrm{m} / \mathrm{z} 11,109+/-5$. The dotted red line corresponds to a multiple of the average noise in the spectrum. It is used as a threshold for the intensity in peak detection. (A) KPC-positive K. pneumoniae strain with peak for the pKpQIL plasmid. (B) KPC-negative strain. In the detection window only noise below the detection threshold can be observed.

and Mueller-Hinton-Agar (MEUS, Piove di Sacco, Italy) were acquired after 24,48 , and $72 \mathrm{~h}$ of incubation and then compared to each other.

Since the intensity of the KPC related peak in some cases is low, we also investigated whether the sensitivity of the automated detection could be enhanced measuring the samples in duplicates. For all samples, spectra were recorded from two spots.

In case of discrepancies between results of the novel MALDI approach and results of routine methods, the samples underwent molecular investigation by GeneXpert ${ }^{\circledR}$ Carba-R (Cepheid, Sunnyvale, CA, United States).

\section{Application Directly to Positive Blood Cultures}

The automated KPC detection was applied to 204 consecutive blood cultures positive for $K$. pneumoniae in routine diagnostics in Bologna. Among those, $n=90$ were KPC-producers, $n=12 \mathrm{M} \beta \mathrm{L}$-producers, $n=1$ was NDM+OXA-48 producer, while $n=101$ were susceptible to carbapenems (MIC of
TABLE 1 | Overview of $K$. pneumoniae isolates from Italy and Germany included in this study.

\begin{tabular}{lccc}
\hline & $\begin{array}{c}\text { Italy } \\
\text { [n] }\end{array}$ & $\begin{array}{c}\text { Germany } \\
\text { [n] }\end{array}$ & $\begin{array}{c}\text { Total } \\
\text { [n] }\end{array}$ \\
\hline KPC & 2386 & 4 & 2390 \\
MBL & 119 & 60 & 179 \\
OXA-48 & 13 & 19 & 32 \\
Carbapenem-resistant & 207 & 14 & 221 \\
non-carbapenemase producers & & & \\
Carbapenem-susceptible & 105 & 3282 & 3387 \\
Total & 2830 & 3379 & 6209 \\
\hline
\end{tabular}

carbapenems lower than epidemiological cut-off). KPCproducers were identified by KPC K-Set, (CorisBioConcept, Gembloux, Belgium), M $\beta L$-producers were identified by KPC+MBL Confirm ID Pack, ROSCO Diagnostika, Taastrup, Denmark), while the strain NDM+OXA-48 was characterized by KPC+MBL Confirm ID Pack, ROSCO Diagnostika, Taastrup, 
Denmark and GeneXpert ${ }^{\circledR}$ Carba-R (Cepheid, Sunnyvale, CA, United States).

The blood specimens were collected in BD BACTEC ${ }^{\mathrm{TM}}$ blood culture media (Plus Aerobic/F, Plus Anerobic/F, Lytic/10 Anaerobic/F and Peds Plus $\left.{ }^{\mathrm{TM}} / \mathrm{F}\right)$, and incubated in a BACTEC ${ }^{\mathrm{TM}}$ FX blood culture system (Becton, Dickinson and Company, Sparks, MD 21152, Benex Limited Shannon, County Clare, Ireland). After the bottles were flagged as positive, the routine workflow at the time of the study provided the species identification by MALDI-TOF MS after a subculturing step on chocolate agar of the enriched pellet obtained from $8 \mathrm{ml}$ of blood culture.

For this study, the blood culture flasks were directly processed with the Sepsityper kit according to instructions of the manufacturer (Bruker Daltonik GmbH, Bremen, Germany) and the extracted bacterial pellet was used to acquire the MALDI spectra.

The KPC subtyping was performed by the MALDI Biotyper software simultaneously with the species identification.

The same pellet was further used to perform STAR-Carba assay (Bruker Daltonik $\mathrm{GmbH}$ ) for carbapenemase confirmation.

\section{Confirmation of Carbapenemase Activity by STAR-Carba Assay}

A subset of $n=243$ randomly chosen (a representative proportion of strains for each year, selected at the discretion of the operator by manually choosing non-juxtaposed strains directly from the strains collection) KPC-producing strains, and all positive blood cultures containing K. pneumoniae $(n=204)$ underwent the MALDI-TOF MS based STAR-Carba imipenem hydrolysis assay (Bruker Daltonik GmbH, Bremen, Germany) to verify the carbapenemase activity. The test was performed according to the manufacturer's instructions. Briefly, $1 \mu l$ loop of bacteria (samples and E.coli ATCC 25922 as negative control, and a PCR-confirmed KPC-producing E. coli as positive control) were resuspended in $50 \mu \mathrm{l}$ of imipenem solution. After $30 \mathrm{~min}$ of incubation at $37^{\circ} \mathrm{C}$ under agitation, the bacteria were pelleted by centrifugation $(2 \mathrm{~min}$ at $14000 \mathrm{rpm}$ ), and $1 \mu l$ of the supernatant was spotted in duplicates onto a MALDI target. Open air dried spots were overlaid with CHCA matrix containing an internal standard. The strains were detected as hydrolyzing or non-hydrolyzing by measuring the intensity of the peak corresponding to the intact form of imipenem $(300 \mathrm{~m} / \mathrm{z})$, normalized on the intensities values of the imipenem peak measured in the negative and positive controls.

\section{RESULTS}

\section{Optimization of the Algorithm for Automated KPC Detection With PCR Confirmed Strains}

Internal calibration of the acquired MALDI-TOF mass spectra and optimization of the newly developed algorithm finally allowed narrowing down the detection window for the peak specific of the bla $a_{\mathrm{KPC}}$-carrying $\mathrm{pKpQIL}$ plasmid to a more specific range of $\mathrm{m} / \mathrm{z} 11,109 \pm 5$ (from initially \pm 15 ).

The novel algorithm detected the bla $a_{\mathrm{KPC}}$-carrying $\mathrm{pKpQIL}$ plasmid related peak in 99/152 PCR confirmed strains (65.1\%).

The visual inspection of these spectra confirmed the presence of the peak in the 99 strains classified as positive by the algorithm (sensitivity 100\%), while the remaining 53 strains didn't show the peak, suggesting that they likely harbor a different $b l a_{\mathrm{KPC}^{-}}$ carrying plasmid.

The KPC peak was not detected by the algorithm in the $n=114$ KPC-PCR negative K. pneumoniae isolates (specificity 100\%).

\section{Evaluation of the Automated KPC Detection With a Large Collection of Strains}

The pKpQIL plasmid-related peak was detected by Biotyper software in overall 2035 of the $n=2390$ KPC-producing isolates $(85.1 \%)$. It was detected in none of the strains resulted positive for the production of a class B or D carbapenemase $(n=179$ $\mathrm{M} \beta \mathrm{L}, n=32$ OXA-48-family), in none of the carbapenemsusceptible isolates $(n=3387)$, but in 5 out of the $n=221$ strains resulted resistant to carbapenems but negative to routine testing for carbapenemase production. The positive predictive value and the negative predictive value resulted $100 \%$ and $91.5 \%$, respectively.

In these 5 strains, the molecular test confirmed the presence of bla $a_{\mathrm{KPC}}$.

With regard to the proportion of KPC-producing strains showing the peak among all KPC-producing isolates, a constantly increasing trend from $63.2 \%$ in the years $2010-2011$ to $94.1 \%$ in 2017 could be observed (Figure 3).

\section{Evaluation of Stability and Reliability of KPC-Peak Detection}

The evaluation of the KPC-detection with this huge set of spectra from clinical strains also comprised the investigation of culture conditions. No performance differences were observed in relation to culture medium used nor to time of incubation.

In $1798 / 2035$ strains, and for $88.4 \%(1798 / 2035)$, the peak was detected in both spots. In $11.6 \%(237 / 2035)$ of the cases, the peak was detected only in one of the two spots.

\section{Application Directly to Positive Blood Cultures}

To assess the further clinical potential, the automated KPCdetection was also applied directly on blood cultures. Among the spectra recorded from the bacterial pellet extracted by Sepsityper, the KPC-specific peak was detected in 83/90 (92.2\%) of the KPCproducing strains, corresponding to $83 / 84$ (98.8\%) of the strains in which the peak was present.

The peak was detected in none of the non-KPC strains $(100 \%$ specificity). 


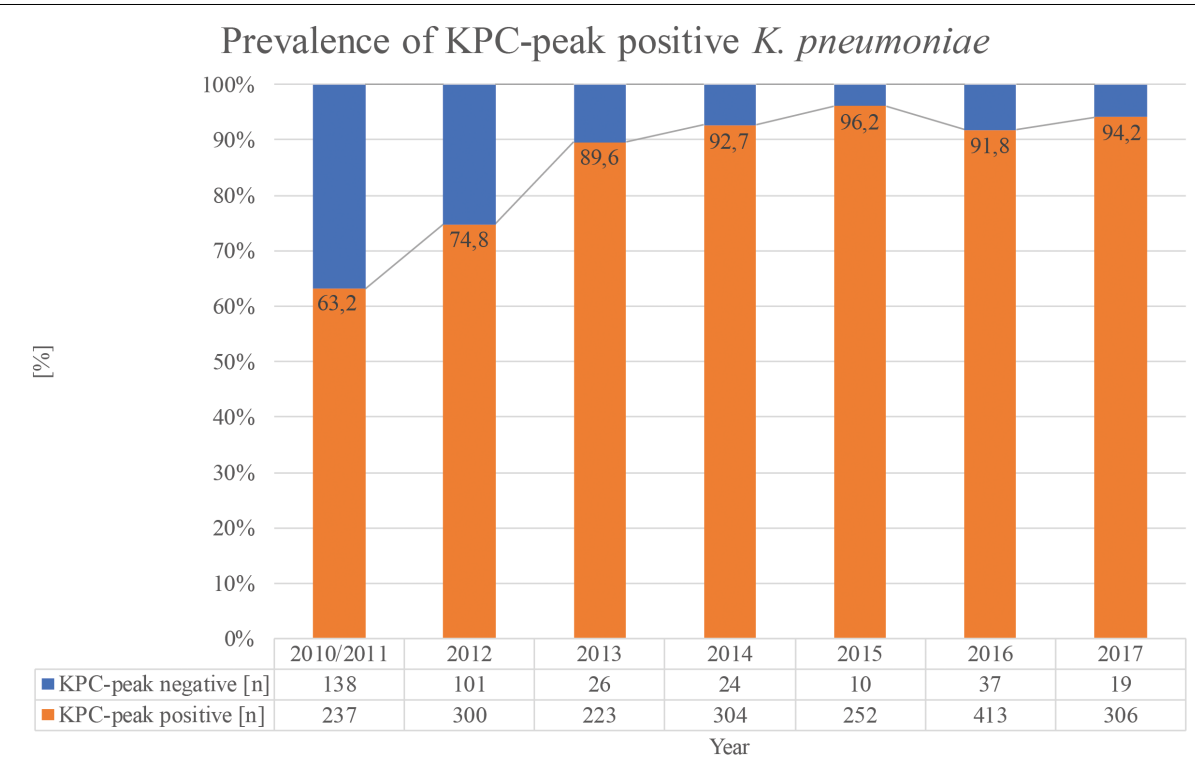

FIGURE 3 | Trend for the years 2010-2017 of KPC-producing K. pneumoniae strains isolated in Bologna showing the pKpQIL plasmid-related peak.

\section{Confirmation of Carbapenemase Activity by STAR-Carba Assay}

STAR-Carba hydrolysis assay resulted positive for all the randomly chosen KPC-producing strains $(n=243)$.

It also resulted positive for all the KPC-producing strains from positive blood cultures $(n=90)$, as well as for all the other carbapenemase-producing strains $(n=12 \mathrm{M} \beta \mathrm{L}$-producers, and $n=1$ NDM+OXA-48-producer), and negative for the $n=101$ carbapenem-susceptible strains from the blood cultures (sensitivity and specificity 100\%).

\section{DISCUSSION}

Carbapenem-resistant enterobacteria are continuously spreading worldwide, and pose a serious threat to public health (Tzouvelekis et al., 2012; Nordmann, 2014). Carbapenemase-producing Enterobacteriaceae (CPE) represent an important cause of acquired infections in hospital settings, burdened by a high mortality rate (Vincent et al., 2009). Although the carbapenemases associated with Enterobacterales show a different prevalence in the different geographic areas, the KPC family has the most extensive global distribution, and it is the most significant (Munoz-Price et al., 2013; van Duin and Doi, 2017).

Rapid methods to detect KPC-producing strains are highly demanded, both for therapeutic and infection control purposes. Several methods are commercially available, relying on different principles, but overall they are either expensive, or slow, with time to report up to $24 \mathrm{~h}$ (Lutgring and Limbago, 2016; Tamma et al., 2017). Recently, a specific peak at $11,109 \mathrm{~m} / \mathrm{z}$, related to a pKpQIL plasmid carrying bla $a_{\mathrm{KPC}}$ was discovered in the MALDITOF mass spectra of KPC-producing Klebsiella pneumoniae (Lau et al., 2014). Different approaches to seek for this peak have been described (Lau et al., 2014; Gaibani et al., 2016; Youn et al., 2016). Nevertheless all of them required a second step after routine species identification process like visual inspection or an additional software analysis.

In this study, we developed a fully MALDI-TOF MS based approach to detect KPC-producing K. pneumoniae strains. This novel approach relies on the indirect identification of bla $a_{\mathrm{KPC}}$-carrying isolates by a now automated detection of the pKpQIL plasmid-related peak at $11,109 \mathrm{~m} / \mathrm{z}$ during the standard routine identification process. The detection of the KPC-related peak is depicted in the MALDI Biotyper system as "presumptive KPC" in the subtyping column. Furthermore, we combined it with a subsequent verification of the carbapenemase activity by an imipenem hydrolysis assay, using the commercially available STAR-Carba kit, and according to the MALDI Biotyper software module (Bruker Daltonik, Bremen). The method was investigated on a large collection of strains from Italy and Germany - two countries with a very different epidemiology of CPE. The study aimed to evaluate this novel approach in a setting close to diagnostic routine, covering a broad period of time (2009-2017), in order to uncover pitfalls which can be missed by smaller dedicated study.

In addition, the integration of the algorithm for instant detection of the KPC-related peak into the MALDI Biotyper RUO software simplifies routine usage as no further special knowledge or additional tools are needed by the operator. The sensitivity of this automated method was found to be $85.1 \%$. Our findings showed that it is mainly related to the prevalence of the KPC-producing strains harboring the $\mathrm{pKpQIL}$ plasmid among all the KPC-producing circulating strains. The visual inspection of the spectra included into the evaluation dataset of this study proved that the automated software detected the $11,109 \mathrm{~m} / \mathrm{z}$ peak in all the strains in which it was present. For 
all strains in which the peak at $11,109 \mathrm{~m} / \mathrm{z}$ was not detected by the automated subtyping algorithm, visual inspection of spectra confirmed this result. Moreover, a permanently increasing trend of the KPC-producing strains showing the pKpQIL specific peak was observed (from $60.2 \%$ in $2010-2011$ to $95.6 \%$ in 2017), suggesting that the spread of KPC in Italy seems to be related to the expansion of this specific clone over the years. Although only 2/4 (50\%) of the German KPC-producing strains showed the $11,109 \mathrm{~m} / \mathrm{z}$ peak, this number is too low to draw conclusions about the prevalence of KPC in German strains. The method showed excellent specificity and positive predictive value $(100 \%)$, as the software correctly classified all non-KPC carbapenemase-producers $(n=179 \mathrm{M} \beta \mathrm{L}$ and $n=32$ OXA48 family), as well as all carbapenem-susceptible isolates as KPC-peak negative. Among the strains resistant to carbapenems but negative for carbapenemase production in routine testing, 216/221 were classified as KPC-peak negative. In $n=5$ strains the peak was detected, and for all of them the molecular testing confirmed the presence of bla KPC. Hence, interestingly, the method in study proved to be able to deliver an earlier detection of KPC carbapenemase in comparison with the other phenotypic methods applied. No differences were observed in the performance of the automated detection using different culture media (blood agar and chromogenic selective and nonselective media), or different incubation times (24-48-72 h), while the use of the spotting in duplicates enabled to achieve a better sensitivity in comparison to single spotting $(85.1 \%$ vs. $80.2 \%)$. The failed detection of the peak is likely due to reasons linked to the sample preparation (operator technical handling, mucousity of the bacterial colonies). Therefore, spotting in duplicate might be advisable for increased robustness and sensitivity as minor biological or technical variations could be compensated.

Since the pKpQIL plasmid-related peak at $11,109 \mathrm{~m} / \mathrm{z}$ is very well detectable in spectra of extractions, our study additionally investigated the performance of the method on positive blood cultures. Compared to colony material, the sensitivity of the peak detection on bacterial pellets extracted directly from positive blood culture using the Sepsityper protocol was $98.8 \%$ with an excellent specificity of $100 \%$. This again underlines the value for clinical routine.

STAR-Carba imipenem hydrolysis assay was used with a subset of strains for confirmation of the carbapenemase activity, both in peak-positive and -negative KPC-producing isolates, and negative controls. It showed $100 \%$ sensitivity and specificity, from colonies as well as from the bacterial pellet extracted from positive blood cultures. In the approach we present here, this assay could play a fundamental role to detect carbapenemase activity, complementary to, and strengthening KPC peak detection. It can be used to detect carbapenemase activity in KPC-producing strains that don't exhibit the presence of the KPC-related peak at $11,109 \mathrm{~m} / \mathrm{z}$, and in all the other carbapenemases of other classes. Further, it can be used as a functional verification of the enzymatic activity predicted by the presence of the KPC-marker detected by subtyping.

Our study shows that the novel MALDI-based approach enabled a reliable and robust detection of KPC-producing
K. pneumoniae isolates, using the same MALDI Biotyper platform as for classical bacterial species identification, with a turnaround time from $10-15 \mathrm{~min}$ to $1.5 \mathrm{~h}$. This method, applicable to colony material as well as directly on positive blood cultures, is unique in performing real time detection of an antibiotic resistance marker in parallel to species identification. It might represent a very useful tool for an early warning for KPC-producing strains, helping to significantly accelerate the proper initiation or change of therapeutic and infection control measures in the future. In comparison to previously described methods based on the $11,109 \mathrm{~m} / \mathrm{z}$ peak detection, the approach we developed presents several advantages. First, the peak detection here is implemented into a commercially available software, and totally automated. Thereby, it doesn't require any operator specific skills regarding spectra processing and analysis. The STAR Carba assay is easy to use, and requires a minimum handling time. Moreover, the interpretation of results is also automated, and hence free from any operatordepending factors, and results are automatically stored and available for eventual future investigation. Thus, this approach can fit with any setting, including high throughput routine laboratories.

The limitation of the presented approach is that the KPC detection by subtyping depends on the association of the peak to bla KPC gene. Partridge (2014) pointed out that BLAST searches indicated that the underlying p019 protein has only been found in plasmids carrying bla $a_{\mathrm{KPC}}$ but an independent genetic movement of the p019 gene might be possible in the future and then impair this indirect detection method. A further limitation is that the sensitivity of the subtyping approach depends on the regional epidemiology of KPC-producing strains, i.e., on the prevalence of the KPCproducing clones harboring the pKpQIL plasmid. Nevertheless, the excellent specificity, and the seamless integration into the commercial software already used for bacterial species identification, might make the implementation of this approach in routine valuable, regardless the current epidemiological context.

Further, as KPC-producing strains have started appearing also among other genera and species of enterobacteria, a future expansion of this approach to other species might be possible.

\section{DATA AVAILABILITY}

The authors state that datasets are available on request. The raw data supporting the conclusion of this manuscript, will be made available by the authors, without undue reservation, to any qualified researcher.

\section{AUTHOR CONTRIBUTIONS}

$\mathrm{MC}, \mathrm{MK}$, and $\mathrm{ABP}$ designed the study. MC, MK, and $\mathrm{ABP}$ carried out the study. $\mathrm{MB}$ and $\mathrm{JG}$ worked on the computer algorithms and statistical analysis together with $\mathrm{MC}$ and $\mathrm{ABP}$. MC, MK, ABP, and SA wrote the manuscript. 


\section{REFERENCES}

Bialvaei, A. Z., Kafil, H. S., Asgharzadeh, M., Yousef Memar, M., and Yousefi, M. (2016). Current methods for the identification of carbapenemases. J. Chemother. 28, 1-19. doi: 10.1179/1973947815Y.0000000063

Burckhardt, I., and Zimmermann, S. (2011). Using matrix-assisted laser desorption ionization-time of flight mass spectrometry to detect carbapenem resistance within 1 to 2.5 h. J. Clin. Microbiol. 49, 3321-3324. doi: 10.1128/JCM.00 287-11

Chen, L., Mathema, B., Chavda, K. D., DeLeo, F. R., Bonomo, R. A., and Kreiswirth, B. N. (2014). Carbapenemase-producing Klebsiella pneumoniae: molecular and genetic decoding. Trends Microbiol. 22, 686-696. doi: 10.1016/j.tim.2014.09.003

Cuzon, G., Naas, T., Truong, H., Villegas, M. V., Wisell, K. T., Carmeli, Y., et al. (2010). Worldwide diversity of Klebsiella pneumoniae that produce betalactamase blaKPC-2 gene. Emerg. Infect. Dis. 16, 1349-1356. doi: 10.3201/ eid1609.091389

Doumith, M., Findlay, J., Hirani, H., Hopkins, K. L., Livermore, D. M., Dodgson, A., et al. (2017). Major role of pKpQIL-like plasmids in the early dissemination of KPC-type carbapenemases in the UK. J. Antimicrob. Chemother. 72, 2241-2248. doi: 10.1093/jac/dkx141

Gaibani, P., Galea, A., Fagioni, M., Ambretti, S., Sambri, V., and Landini, M. P. (2016). Evaluation of matrix-assisted laser desorption ionization-time of flight mass spectrometry for identification of KPC-Producing Klebsiella pneumoniae. J. Clin. Microbiol. 54, 2609-2613. doi: 10.1128/JCM.01242-16

Hoyos-Mallecot, Y., Riazzo, C., Miranda-Casas, C., Rojo-Martin, M. D., Gutiérrez-Fernández, J., and Navarro-Marí, J. M. (2014). Rapid detection and identification of strains carrying carbapenemases directly from positive blood cultures using MALDI-TOF MS. J. Microbiol. Methods 105, 98-101. doi: 10. 1016/j.mimet.2014.07.016

Hrabák, J., Chudácková, E., and Walková, R. (2013). Matrix-assisted laser desorption ionization-time of flight (maldi-tof) mass spectrometry for detection of antibiotic resistance mechanisms: from research to routine diagnosis. Clin. Microbiol. Rev. 26, 103-114. doi: 10.1128/CMR.00058-12

Johansson, Å, Ekelöf, J., Giske, C. G., and Sundqvist, M. (2014). The detection and verification of carbapenemases using ertapenem and matrix-assisted laser desorption ionization-time of flight mass spectrometry. BCM Microbiol. 14:89. doi: 10.1186/1471-2180-14-89

Jung, J. S., Popp, C., Sparbier, K., Lange, C., Kostrzewa, M., and Schubert, S. (2014). Evaluation of matrix-assisted laser desorption ionization-time of flight mass spectrometry for rapid detection of b-lactam resistance in Enterobacteriaceae derived from blood cultures. J. Clin. Microbiol. 52, 924-930. doi: 10.1128/JCM. 02691- 13

Kumarasamy, K. K., Toleman, M. A., Walsh, T. R., Bagaria, J., Butt, F., Balakrishnan, R., et al. (2010). Emergence of a new antibiotic-resistance mechanism in India, Pakistan and the UK: a molecular, biological, and epidemiological study. Lancet Infect. Dis. 10, 597-602. doi: 10.1016/S14733099(10)70143-2

Lau, A. F., Wang, H., Weingarten, R. A., Drake, S. K., Suffredini, A. F., Garfield, M. K., et al. (2014). A rapid matrix-assisted laser desorption ionizationtime of flight mass spectrometry-based method for single-plasmid tracking in an outbreak of carbapenem-resistant Enterobacteriaceae. J. Clin. Microb. 52, 2804-2812. doi: 10.1128/JCM.00694-14

Lutgring, J. D., and Limbago, B. M. (2016). The problem of carbapenemaseproducing-carbapenem-resistant-Enterobacteriaceae detection. J. Clin. Microbiol. 54, 529-534. doi: 10.1128/JCM.02771-15

Munoz-Price, L. S., Poirel, L., Bonomo, R. A., Schwaber, M. J., Daikos, G. L., Cormican, M., et al. (2013). Clinical epidemiology of the global expansion of Klebsiella pneumoniae carbapenemases. Lancet Infect. Dis. 13, 785-796. doi: 10.1016/S1473-3099(13)70190-7

Munoz-Price, L. S., and Quinn, J. P. (2009). The spread of Klebsiella pneumoniae carbapenemase: a tale of strains, plasmids, and transposons. Clin. Infect. Dis. 49, 1739-1741. doi: 10.1086/648078

Nordmann, P. (2014). Carbapenemase-producing Enterobacteriaceae: overview of a major public health challenge. Med. Mal. Infect. 44, 51-56. doi: 10.1016/j. medmal.2013.11.007

Nordmann, P., Dortet, L., and Poirel, L. (2012a). Carbapenem resistance in Enterobacteriaceae: here is the storm! Trends Mol. Med. 18, 263-272. doi: 10. 1016/j.molmed.2012.03.003
Nordmann, P., Gniadkowski, M., Giske, C. G., Poirel, L., Woodford, N., Miriagou, V., et al. (2012b). Identification and screening of carbapenemaseproducing Enterobacteriaceae. Clin. Microbiol. Infect. 18, 432-438. doi: 10.1111/ j.1469-0691.2012.03815.x

Nordmann, P., and Poirel, L. (2014). The difficult-to-control spread of carbapenemase producers in Enterobacteriaceae worldwide. Clin. Microbiol. Infect. 20, 821-830. doi: 10.1111/1469-0691.12719

Osei Sekyere, J., Govinden, U., and Essack, S. Y. (2015). Review of established and innovative detection methods for carbapenemase-producing gram-negative bacteria. J. Appl. Microbiol. 119, 1219-1233. doi: 10.1111/jam.12918

Partridge, S. R. (2014). Tn4401 carrying blaKPC is inserted within another insertion in pKpQIL and related plasmids. J. Clin. Microbiol. 52, 4448-4449. doi: 10.1128/JCM.02426-14

Pranada, A. B., Witt, E., Bienia, M., Kostrzewa, M., and Timke, M. (2017). Accurate differentiation of mycobacterium chimaera from mycobacterium intracellulare by MALDI-TOF MS analysis. J. Med. Microbiol. 66, 670-677. doi: 10.1099/jmm. 0.000469

Rapp, E., Samuelsen, $\varnothing$, and Sundqvist, M. (2018). Detection of carbapenemases with a newly developed commercial assay using matrix assisted laser desorption ionization-time of flight. J. Microbiol. Methods 146, 37-39. doi: 10.1016/j.mimet. 2018.01.008

Rood, I. G. H., and Li, Q. (2017). Review: molecular detection of extended spectrum- $\beta$-lactamase- and carbapenemase-producing Enterobacteriaceae in a clinical setting. Diagn. Microbiol. Infect. Dis. 89, 245-250. doi: 10.1016/j. diagmicrobio.2017.07.013

Sakarikou, C., Ciotti, M., Dolfa, C., Angeletti, S., and Favalli, C. (2017). Rapid detection of carbapenemase-producing Klebsiella pneumoniae strains derived from blood cultures by matrix-assisted laser desorption ionization-time of flight mass spectrometry (MALDI-TOF MS). BMC Microbiol. 17:54. doi: 10.1186/ s12866-017-0952-3

Sparbier, K., Schubert, S., Weller, U., Boogen, C., and Kostrzewa, M. (2012). Matrixassisted laser desorption ionization-time of flight mass spectrometry-based functional assay for rapid detection of resistance against beta lactam antibiotics. J. Clin. Microbiol. 50, 927-937. doi: 10.1128/JCM.05737-11

Tamma, P. D., Opene, B. N., Gluck, A., Chambers, K. K., Carroll, K. C., and Simner, P. J. (2017). Comparison of 11 phenotypic assays for accurate detection of carbapenemase-producing Enterobacteriaceae. J. Clin. Microbiol. 55, 1046-1055. doi: 10.1128/JCM.02338-16

Tzouvelekis, L. S., Markogiannakis, A., Psichogiou, M., Tassios, P. T., and Daikos, G. L. (2012). Carbapenemases in Klebsiella pneumoniae and other Enterobacteriaceae: an evolving crisis of global dimensions. Clin. Microbiol. Rev. 25, 682-707. doi: 10.1128/CMR.05035-11

van Duin, D., and Doi, Y. (2017). The global epidemiology of carbapenemaseproducing Enterobacteriaceae. Virulence 8, 460-469. doi: 10.1080/21505594. 2016.1222343

Vincent, J. L., Rello, J., Marshall, J., Silva, E., Anzueto, A., Martin, C. D., et al. (2009). International study of the prevalence and outcomes of infections in intensive care units. JAMA 302, 2323-2329. doi: 10.1001/jama.2009.1754

Youn, J.-H., Drake, S. K., Weingarten, R., Frank, K. M., Dekker, J. P., and Lau, A. F. (2016). Clinical performance of a matrix-assisted laser desorption ionization-time of flight mass spectrometry method for detection of certain blaKPC-containing plasmids. J. Clin. Microbiol. 54, 35-42. doi: 10.1128/JCM. 01643-15

Conflict of Interest Statement: MK and JG are employees of Bruker Daltonik $\mathrm{GmbH}$, the manufacturer of the MALDI Biotyper system.

The remaining authors declare that the research was conducted in the absence of any commercial or financial relationships that could be construed as a potential conflict of interest.

Copyright (c) 2018 Cordovana, Kostrzewa, Glandorf, Bienia, Ambretti and Pranada. This is an open-access article distributed under the terms of the Creative Commons Attribution License (CC BY). The use, distribution or reproduction in other forums is permitted, provided the original author(s) and the copyright owner(s) are credited and that the original publication in this journal is cited, in accordance with accepted academic practice. No use, distribution or reproduction is permitted which does not comply with these terms. 